\title{
Using DSMs for the Visualization and Analysis of Decision Models in Business Processes
}

\author{
Dario Campagna ${ }^{1}$, Carlos Kavka $^{1}$, Sara Nicastro ${ }^{1}$, Carlo Poloni ${ }^{2}$, Alessandro Turco ${ }^{1}$ \\ ${ }^{1}$ ESTECO SpA, Trieste, Italy \\ ${ }^{2}$ Department of Engineering and Architecture, University of Trieste, Trieste, Italy
}

\begin{abstract}
Business processes are represented nowadays with industry standards like Business Process Model and Notation (BPMN) and Decision Model and Notation (DMN). Business analysts can graphically represent the flow of a business process with BPMN. At the same time, they can use DMN to model the logic of decisions and display their requirements. These graphical standards help in managing the complexity of typical business processes and decisions. Anyway, a business analyst may end up with models that are difficult to comprehend, to analyze and to maintain. In this paper we present a novel technique to visualize the Decision Requirement Graph (DRG) of a DMN model with a DSM. The DSM representation gives to a business analyst a compact and concise view of the whole DRG. Cluster analysis of the resulting DSM can provide alternative visualization of the DRG and contribute to a better understanding of the decisions behind a business process.
\end{abstract}

Keywords: Decision Modeling, DMN, DRG, DSM, clustering.

\section{Introduction}

The Decision Model and Notation (DMN) Version 1.3 (OMG, 2020) is a standard for decision modeling developed by the Object Management Group (OMG). The purpose of DMN is to provide the constructs that are needed to model decisions (OMG, 2020). A decision model in DMN has two levels, decision requirements and decision logic. The decision requirements level defines the decisions, their interrelationships, and their requirements for decision logic. The decision logic level defines the required decisions in sufficient detail to allow validation and/or automation. In this paper we focus on the decision requirements level of DMN.

The decision requirements level of a decision model in DMN consists of a Decision Requirements Graph (DRG) depicted in one or more Decision Requirements Diagrams (DRDs) (OMG, 2020). As stated in (OMG, 2020): "For any significant domain of decisionmaking a DRD representing the complete DRG may be a large and complex diagram.". A good practice when dealing with a complex DRG is to use many DRDs, with each DRD visualizing a partial view of the DRG. In this way you avoid having a single large and intricate DRD that would be difficult to read and to work with. This practice poses a question: "How can we visualize the DRG as a whole?". Possible answers are: (1) a list of all the DRG elements; (2) a tree-like representation of the DRG. Both answers are valid, however, none of them represents all the dependencies between DRG elements. 
Our answer to the above question is to use a Design Structure Matrix (DSM) (Eppinger and Browning, 2012) to visualize the DRG. The DSM we propose has DRG elements as rows and columns. Off-diagonal marks model requirements. We adopt the Input in Rows/Feedback Above Diagonal (IR/FAD) convention. To the best of our knowledge this is the first time DSMs are used in the context of decision modeling and DMN. In this paper we describe how to create different DSM visualizations for a DRG. Moreover, we show how to generate alternatives DRDs representation using a clustering algorithm.

The paper is organized as follows. Section 2 gives an overview of the decision requirements level of DMN and introduces the example we use throughout the paper. Section 3 describes how to build alternative DSM visualizations for a DRG. In Section 4 we explore the relation between clustered DSMs and DRDs. Section 5 concludes the paper and presents some future research directions.

\section{The DMN Decision Requirements Level}

In this section we present some of the basic concepts of the DMN decision requirements level. We also introduce the DMN example that we use in the following sections.

\subsection{DRG and DRDs}

A DRG models a domain of decision making, showing the most important elements involved in it and the dependencies between them (OMG, 2020). The elements composing the DRG, as defined in (OMG, 2020), are the following.

- A Decision element denotes the act of determining an output from a number of inputs, using decision logic which may reference one or more Business Knowledge Models.

- A Business Knowledge Model (BKM) element denotes a function encapsulating business knowledge, e.g., as business rules, a decision table, or an analytic model.

- An Input Data element denotes information used as an input by one or more Decisions.

- A Knowledge Source element denotes an authority for a Business Knowledge Model or Decision.

- A Decision Service element denotes a set of reusable decisions that can be invoked internally or externally.

Three kinds of requirements express the dependencies between the above elements. They are defined in (OMG, 2020) as follows.

- An Information Requirement denotes Input Data or Decision output being used as input to a Decision.

- A Knowledge Requirement denotes the invocation of a Business Knowledge Model or Decision Service by the decision logic of a Decision. 
- An Authority Requirement denotes the dependence of a DRG element on another DRG element that acts as a source of guidance or knowledge.

As mentioned before, DRG elements and requirements can be depicted in one or more DRD. Table 1 summarizes the notation of the DRD components we use in this paper. For the complete list of components see (OMG, 2020).

Table 1. DRD components.

\begin{tabular}{|c|c|c|}
\hline \multicolumn{2}{|c|}{ Component } & Notation \\
\hline \multirow[t]{4}{*}{ Elements } & Decision & Decision \\
\hline & $\begin{array}{c}\text { Business } \\
\text { Knowledge } \\
\text { Model }\end{array}$ & $\begin{array}{c}\text { Business } \\
\text { Knowledge Model }\end{array}$ \\
\hline & Input Data & Input Data \\
\hline & $\begin{array}{c}\text { Knowledge } \\
\text { Source }\end{array}$ & $\begin{array}{l}\text { Knowledge } \\
\text { Source }\end{array}$ \\
\hline \multirow[t]{3}{*}{ Requirements } & $\begin{array}{l}\text { Information } \\
\text { Requirement }\end{array}$ & \\
\hline & $\begin{array}{l}\text { Knowledge } \\
\text { Requirement }\end{array}$ & -- \\
\hline & $\begin{array}{c}\text { Authority } \\
\text { Requirement }\end{array}$ & -- \\
\hline
\end{tabular}

\subsection{The Originations Example}

Chapter 11 of (OMG, 2020) is dedicated to two DMN examples. The first example is called "Originations" and shows the use of DMN to model and execute decision-making for loan originations. This example is complex enough to show the advantages of the DSM visualization, it fits well in the pages of a paper and it's publicly available. From now on we refer to this example as Originations.

Figure 1 shows the "DRD of all automated decision making" of Originations. This DRD includes all the DRG elements except one (an Input Data that appears only in another DRD). It depicts 30 elements and 46 requirements. It is not that large but certainly intricate.

The DMN specification states that "It might be considered more convenient to draw separate (but overlapping) DRDs [...]" (OMG, 2020) and presents four DRDs. In the next sections we assume that the DMN model was created by drawing only these separate DRDs. For the complete example description see (OMG, 2020). 


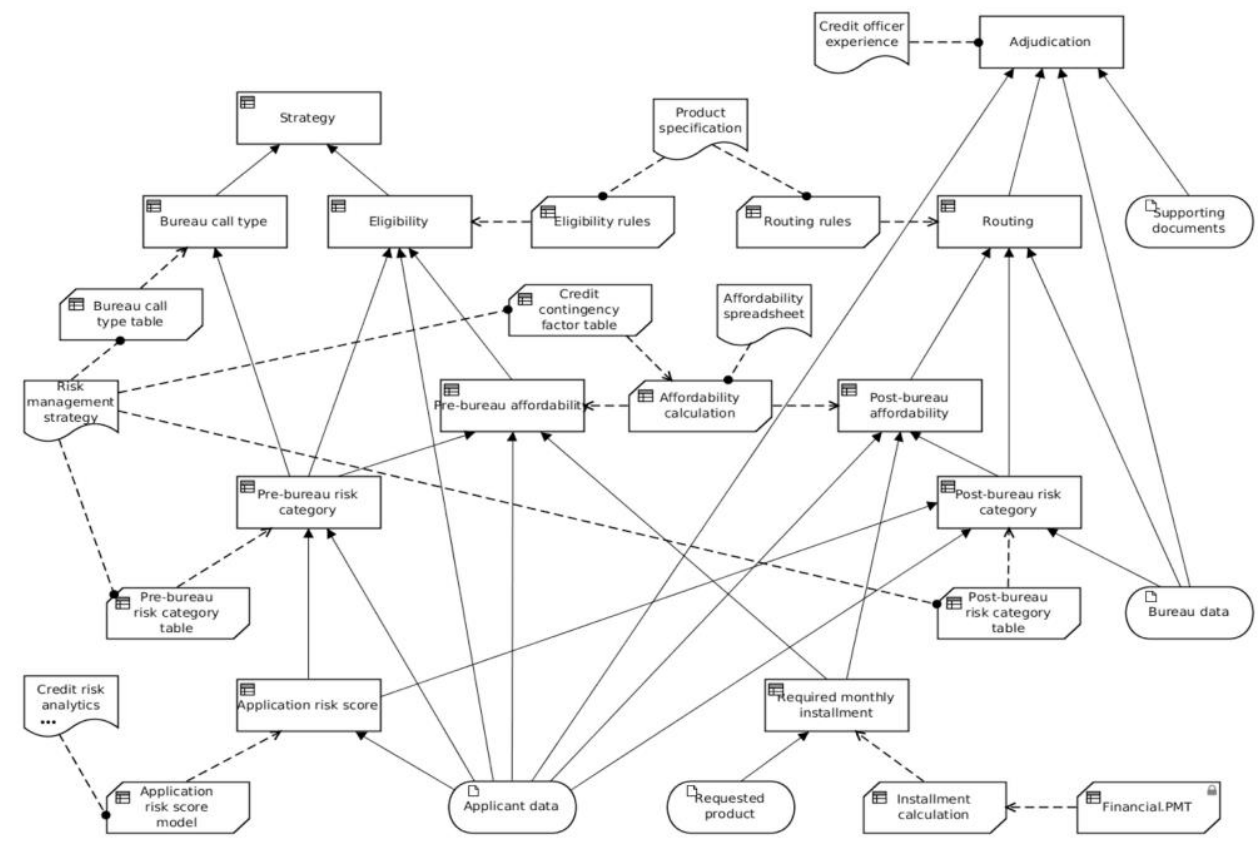

Figure 1: "DRD of all automated decision-making" of the Originations example (OMG, 2020).

\section{DSM for DRG visualization}

A DRG is a graph of DRG elements connected by requirements. In this section we show how to build alternative DSM visualizations for a DRG.

\subsection{From the DRG Graph to a DSM}

The system we want to represent using a DSM is the domain of decision-making modeled by a DRG. DRG elements decompose the domain in subparts, we can view these subparts as the elements comprising our system. As a consequence, the interactions among the system elements are the dependencies between the domain subparts determined by DRG requirements. Given these considerations it is quite straightforward to create a binary DSM (IR/FAD) from a DRG.

- $\quad$ Add a row/column for each DRG element.

- $\quad$ For each DRG requirement, add a mark on the corresponding off-diagonal cell.

By applying the above translation to the DRG of Originations, and then using the Cambridge Advanced Modeller (Wynn et al., 2010) partitioning algorithm, we get the binary DSM shown in Figure 2. We do not have marks above the diagonal. Since the DRG graph of Originations is acyclic we can always find an ordering of rows/columns with no feedback cycles. 


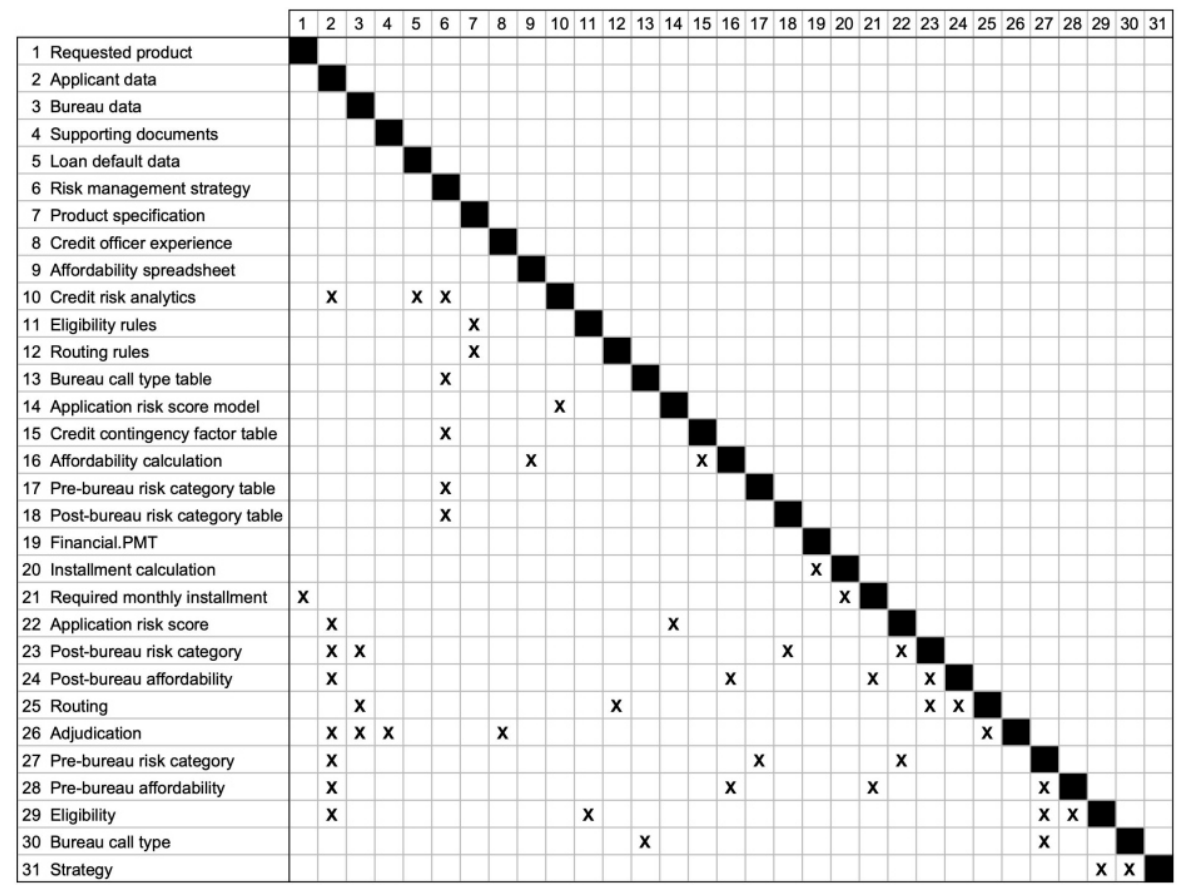

Figure 2: Binary DSM for the DRG of Originations.

The DRG of Originations, with its 31 elements and 49 requirements, is rather simple. Despite this fact the advantages of the DSM visualization are evident. As Figure 2 and Figure 1 suggest, the DSM visualization is more compact and concise than an equivalent DRD, i.e., a graph-based representation with 31 nodes and 49 edges. The DSM visualization of a DRG scales better than a single DRD representation as the number of elements and requirements increase.

Both DRG elements and DRG requirements can be of different kinds. A DRG element can be a Decision, BKM, Knowledge Source or Input Data. A DRG requirement can be an Information Requirement, Knowledge Requirement or Authority Requirement. We can view the kinds of DRG element and DRG requirement as attributes of the system elements and their dependencies. We can use these attributes to extend the binary DSM as shown in Figure 3. We label the system elements accordingly using different colors. We use different symbols in off-diagonal cells to distinguish the three kind of dependencies.

These extensions to the binary DSM serve as visual aids for a person reading the DSM to get insights about the architecture of a DRG. Colors and symbols help in matching DSM elements and interactions to the DRG element and requirements they come from. Together with the DSM improved layout, they allow a clear understanding of the decomposition of the domain of decision-making and make it quick to determine the dependencies of a given 
element. The same task could take more time in an intricate DRD and may be difficult to perform when a single DRD visualizing the whole DRG is not present.

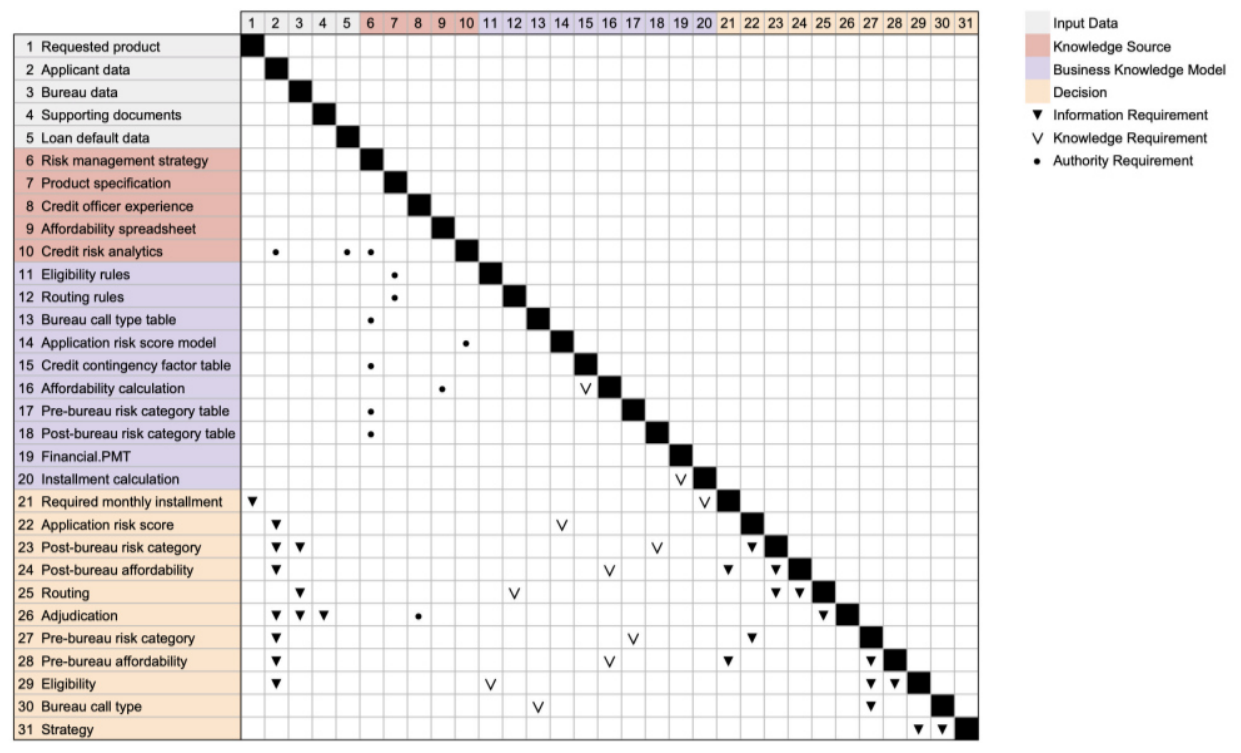

Figure 3: Extended binary DSM for the DRG of Originations.

We informally validated the above considerations about the advantages of a DSM representation of the DRG by getting feedback from decision modeling experts and practitioners. The compactness of the visualization and the time required to get certain information about the DRG are performance indicators that we think are improved by the DSM visualization. Nevertheless, a formal study is needed to consolidate our preliminary observations.

\subsection{Decision Services and DRDs}

The DSM visualization presented in Section 3.1 can be further extended to take into account Decision Services and DRDs. Decision Services denote sets of reusable decisions. DRDs represent sub-graphs of the DRG graph. Both Decision Services and DRDs define partial views of the DRG graph, we can visualize them using the external inputs/outputs convention as shown in Figure 3.

For each Decision Service we add an external column and mark cells as follows: $\square$ for exposed Decisions; $\triangleleft$ for internal Decisions; I for Input Data. For each DRD, we add an external column and mark with an $\mathrm{X}$ all the cells corresponding to elements appearing in the DRD. We color the cells of elements in the DRD that have no outputs.

Figure 3 also shows the two Decision Services of Originations using two clusters. The blue (smaller) cluster is for the "Routing" Decision Service. The green (bigger) cluster is for the "Bureau Strategy" Decision Service. While we can use clusters to model Decision Services 
(and DRDs) we can't cover all possible cases. For example, we cannot use clusters to model three Decision Services that share one element.
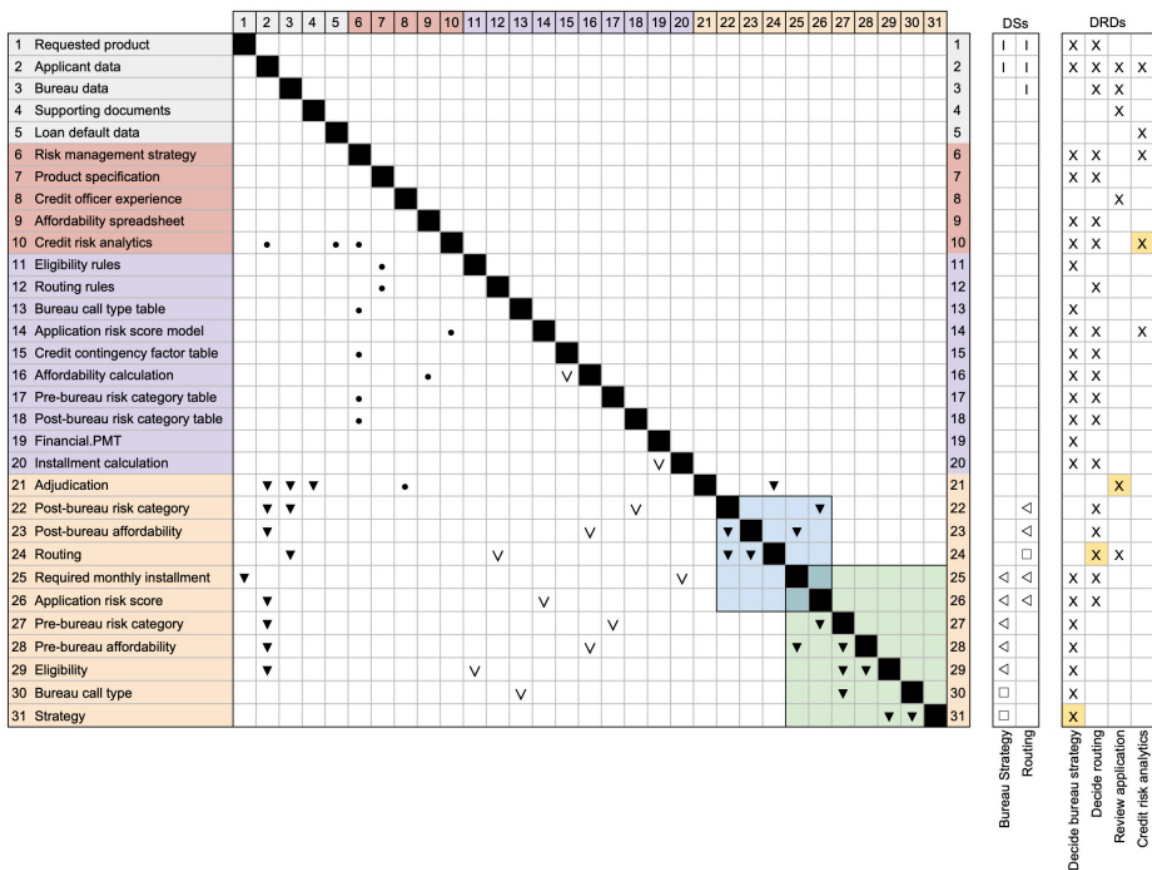

Figure 4: Visualization of Decisions Services and DRDs.

\section{DSM Clustering and DRDs}

Section 3.1 describes how to get a DSM from a DRG. In this section we explore the other way around. That is, how to generate DRDs from a DSM. A typical analysis performed on static DSM is the clustering analysis. The DSM we get from a DRG is a static DSM. We can perform a clustering analysis on it for at least two purposes:

- $\quad$ To generate alternative DRDs.

- $\quad$ To generate DRDs when we have only one big DRD or no DRDs.

Given a clustered DSM we can create DRDs as follows. For each cluster:

1. Create a DRD.

2. Add to the DRD all the elements in the cluster.

3. For each element in the cluster, add to the DRD the input elements that are outside of the cluster. 
To show this translation, we apply the clustering algorithm implemented in (Thebeau, 2001) to the DSM of Originations (Figure 2). We want to get 3 to 5 clusters and would like the algorithm to be consistent across multiple runs. The behavior of the algorithm can be changed by varying the values of 8 parameters, it is possible to let the algorithm ignore specific elements of the DSM and to define weak and strong interaction in the DSM. To check the effects of changing parameters and DSM data we use the likeness clustering analysis implemented in (Thebeau, 2001). Table 2 lists some of the results of our experiments. We use the values reported in (Thebeau, 2001) as default for the parameters. To vary the interaction levels, we set the value 2 for dependencies between Decisions and the value 0.5 for dependencies involving Input Data and Knowledge Sources. All the results of our experiments are available at https://github.com/DSM-2020-paper/dsmclustering-for-dmn.

Table 2. Results of experiments with clustering algorithm.

\begin{tabular}{|c|c|c|c|}
\hline Experiment & $\begin{array}{c}\text { Likeness } \\
\text { mean }\end{array}$ & $\begin{array}{c}\text { Likeness } \\
\text { median }\end{array}$ & $\begin{array}{c}\text { Number of } \\
\text { clusters }\end{array}$ \\
\hline pow_bid=-1 (penalize small \\
clusters) & 0.5749 & 0.50706 & $4-10$ \\
\hline $\begin{array}{c}\text { pow_bid=-1,pow_dep=4 } \\
\text { (emphasize high interactions) }\end{array}$ & 0.53431 & 0.5501 & $5-9$ \\
\hline $\begin{array}{c}\text { pow_bid =-1, exclude } \\
\text { "Applicant data" and "Risk } \\
\text { management strategy" } \\
\text { (elements with many } \\
\text { dependencies) }\end{array}$ & 0.6991 & 0.70922 & $\begin{array}{c}6-10 \\
\text { (excluded } \\
\text { elements } \\
\text { counted as } \\
\text { clusters) }\end{array}$ \\
\hline $\begin{array}{c}\text { pow_bid=-1,times =4, } \\
\text { stable_limit }=4 \text { (run for a } \\
\text { longer time) }\end{array}$ & 0.53119 & 0.5511 & $4-7$ \\
\hline $\begin{array}{c}\text { pow_bid=-1 and varied } \\
\text { interaction levels }\end{array}$ & 0.60985 & 0.61042 & $8-10$ \\
\hline $\begin{array}{c}\text { Varied interaction levels and } \\
\text { emphasize high interactions }\end{array}$ & 0.61692 & 0.6124 & $7-11$ \\
\hline $\begin{array}{c}\text { Emphasize interactions and } \\
\text { exclude elements }\end{array}$ & 0.70994 & 0.70361 & $7-10$ \\
\hline
\end{tabular}

From the results shown in Table 2 we see that as the likeness mean and median increase, the lower bound of the number of clusters increases. We get interesting results with three settings.

- Penalize small clusters: The number of clusters varies from 4 to 10, DSM with 4 to 6 clusters can be used to generate DRDs.

- $\quad$ Exclude elements: The number of clusters for generating DRDs is between 4 and 8 . Small clusters can be ignored when their elements already appear in other DRDs. 
- Run for a longer time: This setting gets useful clustering most of the time. Small clusters can be ignored when their elements already appear in other DRDs.

In the other cases we get too many small clusters (i.e., clusters of size 1 to 3 ). Figure 5 shows a clustered DSM obtained with the settings to exclude elements.

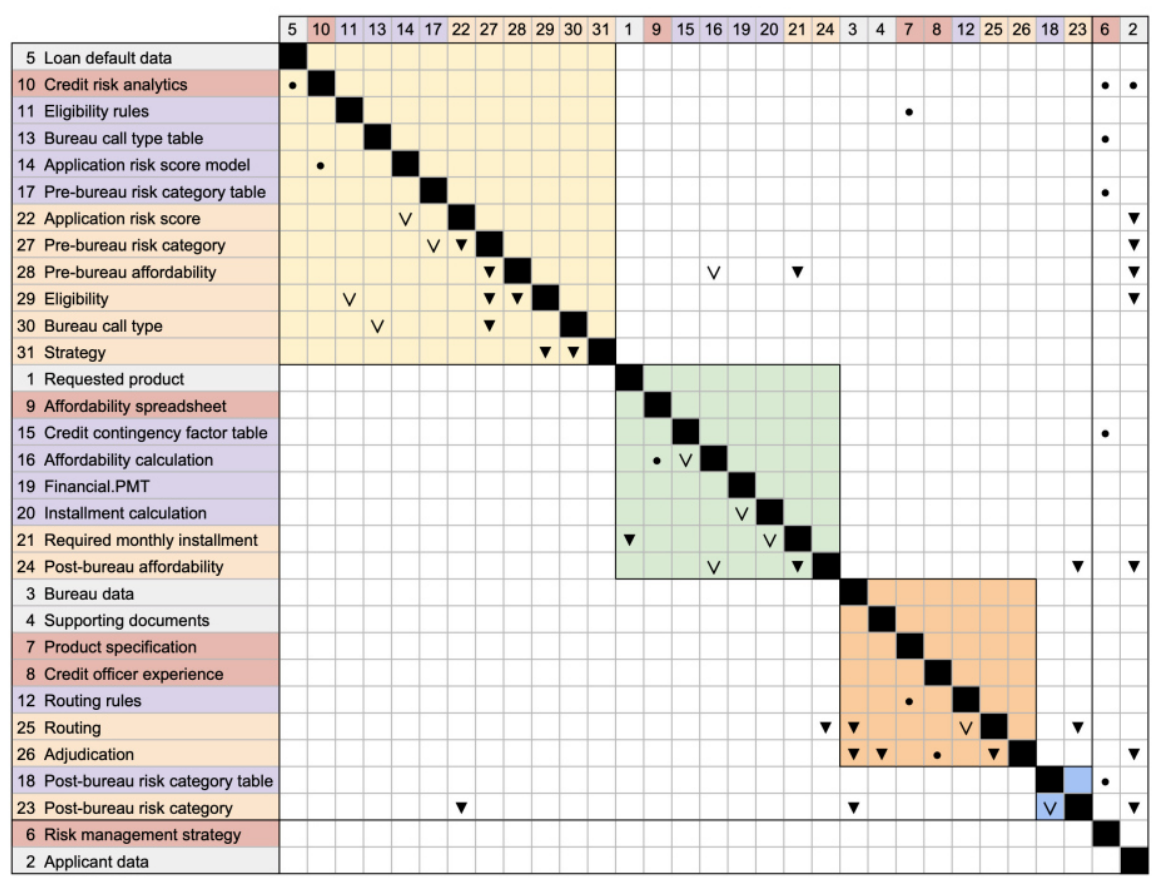

Figure 5: clustered DSM excluding "Applicant data" and "Risk management strategy".

The DSM in Figure 5 has 4 clusters (highlighted with different colors). The clustering algorithm adds back the excluded elements on the outside edges of the clustered DSM. These two elements appear in DRDs with elements requiring them. The fourth cluster can be merged with the second or third cluster. Figure 6 shows the DRD we get from the second cluster. It includes the two excluded elements and the two elements of cluster 4 . The DRD in Figure 6 was created with Cardanit DMN editor (Cardanit, 2020).

The DRDs we get from a clustered DSM can be a good starting point for exploring alternative DRDs representation for a DRG. An interactive tool could use a clustering algorithm to determine the elements in each DRD. The tool could display the generated DRDs using an auto-layout algorithm. The tool could also offer ways to change/merge clusters and immediately see those changes in the DRDs. Moreover, the additional attributes for elements and interaction introduced in Section 3.1 could be used to implement a tailored clustering algorithm for this application. 


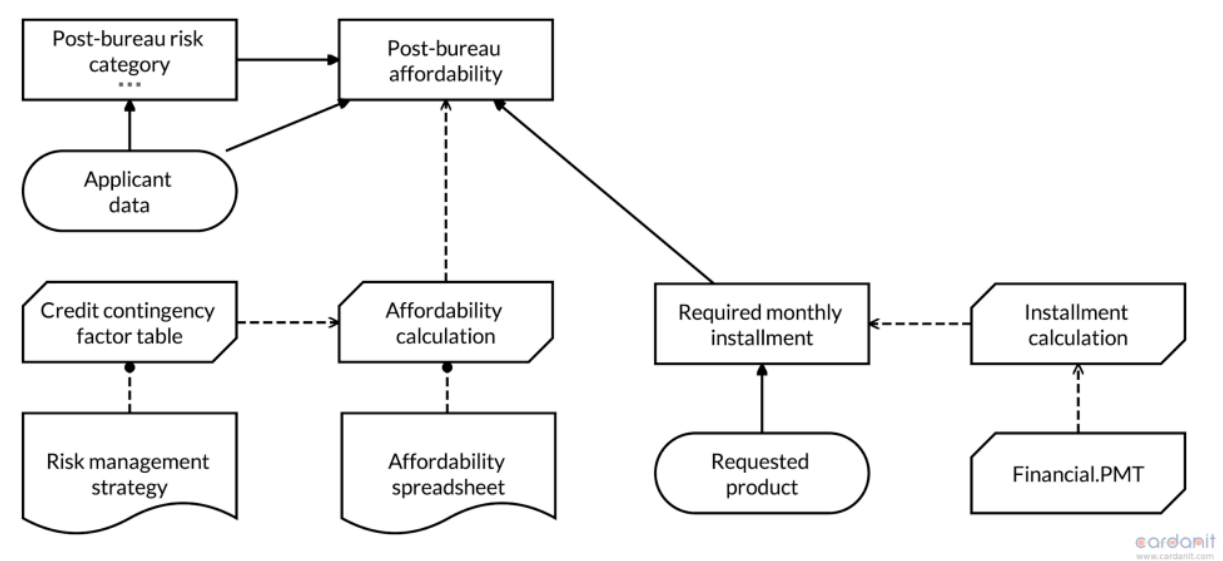

Figure 6: DRD from the second cluster shown in Figure 4.

\section{Conclusions}

In this paper we explored a novel technique to visualize and analyze a decision model using DSMs. We showed how to create different DSM visualizations for a DRG graph and described how to generate alternative DRDs representation of a DRG with clustered DSMs. We used the Originations example described in (OMG, 2020) to demonstrate both the DSM-based visualization and analysis. For the clustering analysis we used the algorithm presented in (Thebeau, 2001). The results presented in this paper suggests that a DSM visualization of a DRG is more compact and concise than an equivalent DRD representation. Moreover, a clustered DSM can be a good starting point for experimenting alternative DRD representations and getting a better understanding of a decision model. Future research work will focus on the implementation of an interactive tool for the DSMbased visualization of DRGs. We also plan to consolidate our preliminary observations about the advantages of the DSM visualization and to study other clustering algorithms for the automatic generation of DRDs.

\section{References}

Wynn D.C., Wyatt D.F., Nair S.M.T. and Clarkson P.J. An Introduction to the Cambridge Advanced Modeller. Proceedings of MMEP 2010. Cambridge, UK.

Cardanit, 2020. https://www.cardanit.com (accessed 27 April 2020).

Eppinger, S.D., Browning, T.R., 2012. Design Structure Matrix Methods and Applications. MIT Press, Cambridge, MA.

OMG, 2020. Decision Model and Notation Version 1.3. https://www.omg.org/spec/DMN/1.3/PDF (accessed 14 April 2020).

Thebeau, R.E., 2001. Knowledge Management of Systems Interfaces for Product Development Processes. https://dsmweborg.files.wordpress.com/2019/05/msc_thebeau.pdf (accessed 14 April 2020).

Contact: Dario Campagna, ESTECO SpA, Padriciano 99, 34149, Trieste, Italy, Phone +39 040 3755548, Fax +39040 3755549, campagna@esteco.com 